\title{
Inhibitory But Not Excitatory Cortical Neurons Require Presynaptic Brain-Derived Neurotrophic Factor for Dendritic Development, as Revealed by Chimera Cell Culture
}

\author{
Keigo Kohara, ${ }^{1,2}$ Akihiko Kitamura, ${ }^{1,2}$ Naoki Adachi, ${ }^{1,2}$ Megumi Nishida, ${ }^{1,2}$ Chiaki Itami, ${ }^{3}$ Shun Nakamura, ${ }^{3}$ and \\ Tadaharu Tsumoto ${ }^{1,2}$ \\ ${ }^{1}$ Division of Neurophysiology, Osaka University Graduate School of Medicine, Suita 565-0871, Japan, ${ }^{2}$ Core Research for Evolutional Science and \\ Technology, Japan Science and Technology Corporation, Kawaguchi 442-0012, Japan, and ${ }^{3}$ Division of Biochemistry and Cellular Biology, National Institute \\ of Neuroscience, Kodaira 187-8502, Japan
}

To address questions of whether endogenous BDNF acts differentially on inhibitory and excitatory neurons, and through what routes, we used chimera culture of cerebral cortical neurons derived from $B D N F-/-$ mice and another type of transgenic mice that express green fluorescence protein and BDNF. Presynaptic BDNF transferred to both types of neurons, GABA-synthesizing enzyme-positive and -negative neurons. The latter neurons were confirmed to be glutamatergic with immunocytochemistry. Dendritic development of the former inhibitory neurons was promoted by endogenous BDNF transferred from presynaptic, excitatory neurons. In contrast, dendritic development of excitatory neurons was not related to the presence or absence of presynaptic BDNF, suggesting that BDNF acts on inhibitory neurons through an anterograde, transsynaptic route so as to promote dendritic development, whereas this is not the case in excitatory neurons.

Key words: neurotrophin; brain-derived neurotrophic factor; dendritic growth; inhibitory neuron; visual cortex; chimera cell culture; green fluorescence protein

\section{Introduction}

Brain-derived neurotrophic factor (BDNF) is known to play a crucial role in development and plasticity of neuronal circuits in the CNS (for review, see Thoenen, 1995; McAllister et al., 1999; Bibel and Barde, 2000; Poo, 2001). In addition to actions on dendritic growth of pyramidal neurons in visual cortex (McAllister et al., 1995, 1996, 1997; Horch et al., 1999), BDNF has been reported to regulate development of inhibitory neurons containing GABA in the brain. For example, BDNF promotes the phenotype differentiation of GABAergic neurons in hippocampus and striatum (Ip et al., 1993; Nawa et al., 1993; Mizuno et al., 1994; Marty et al., 1996; Ivkovic and Ehrlich, 1999; Yamada et al., 2002), facilitates dendritic development of hippocampal GABAergic neurons in culture (Bartrup et al., 1997; VicarioAbejon et al., 1998), increases the density of inhibitory synapses (Marty et al., 2000) or the size of inhibitory terminals (Bolton et al., 2000) of hippocampal neurons, and plays a role in activitydependent regulation of inhibition at cortical and hippocampal

\footnotetext{
Received 0ct. 9, 2002; revised April 21, 2003; accepted April 23, 2003.

This work was supported by a grant-in-aid for Scientific Research on Priority Areas-Advanced Brain Science Project from the Ministry of Education, Science, Sports and Culture of Japan (T.T.). We express many thanks to Drs. M. Okabe, R. Katoh-Semba, T. Kaneko, T. Torashima, Y. Hata, and K. Souya for providing GFP mice, anti-BDNF antibody, anti-glutaminase antibody, and technical advices of immunostaining, drawing neuronal dendrites, and confoca microscopy, respectively.

Correspondence should be addressed to Dr. Tadaharu Tsumoto, Division of Neurophysiology (D-14), 0saka University Graduate School of Medicine, 2-2 Yamadaoka, Suita 565-0871, Japan. E-mail: ttsumoto@nphys.med.osakau.ac.jp.

Copyright $\odot 2003$ Society for Neuroscience $\quad$ 0270-6474/03/236123-09\$15.00/0
}

synapses (Rutherford et al., 1997; Tanaka et al., 1997; Frerking et al., 1998). Furthermore, depletion and overexpression of BDNF in transgenic mice impairs dendritic growth of cerebellar Purkinje cells (Schwartz et al., 1997) and accelerates maturation of GABAergic neurons in visual cortex (Huang et al., 1999), respectively.

Because these results were obtained with exogenously applied BDNF or with the nonphysiological level of BDNF, however, an important question of whether endogenous BDNF in the physiological condition exerts such an action is not answered yet. Also, a route through which endogenous BDNF acts on inhibitory neurons is not clarified, although glutamatergic or catecholaminergic neurons were suggested to be a source of BDNF (Nawa et al., 1995; Altar et al., 1997; Marty et al., 1997; Fawcett et al., 2000). Furthermore, another question of whether endogenous BDNF acts on inhibitory neurons in a different way from its action on excitatory neurons has not explicitly been answered yet.

To address these questions, we took an advantage of neurons derived from BDNF knock-out mice, because in these neurons one can detect an uptake of endogenous BDNF from other neurons of wild-type mice if both types of neurons are cocultured. In this preparation, however, it is practically difficult to differentiate neurons of knock-out mice from those of wild-type mice, because BDNF can be transferred from cell to cell. To overcome this problem, we prepared a mixed cell culture with another type of transgenic mice in which all the cells express green fluorescence protein (GFP) (Okabe et al., 1997; Fujikawa et al., 2000). In this chimera culture of neurons prepared from the different types of 
transgenic mice, we could unambiguously identify neurons having the potential to express endogenous BDNF with GFP tag and those lacking the potential under a fluorescence microscope. Combined with immunocytochemistry with antibody to GABAsynthesizing enzyme, we found that GABAergic neurons require BDNF of presynaptic, excitatory neurons for development of their dendrites, but excitatory neurons do not require such presynaptic BDNF.

\section{Materials and Methods}

Chimera culture of neurons. Neonatal GFP mice (C57BL/6; provided by Dr. M. Okabe, Genome Information Research Center, Osaka University, Suita 565-0871, Japan) and mice (C57BL/6; provided by Regeneron Pharmaceuticals, Tarrytown, NY) that were confirmed to be BDNF-/mice with genotyping were anesthetized with ketamine $(>30 \mathrm{mg} / \mathrm{kg}$, i.p. $)$ and then killed by cervical dislocation at postnatal days $2-3$. Genotyping of neonatal mice was performed in the same way as described previously (Itami et al., 2000). The experimental procedures met the regulation of the Animal Care Committee of Osaka University Graduate School of Medicine. Neurons derived from $B D N F-/-$ and GFP mice were cultured on the same glial feeder layers that had been prepared previously from $B D N F-/-$ mice. The density of GFP and $B D N F-/-$ neurons were $25-50$ cells $/ \mathrm{cm}^{2}$ and $\sim 1000$ cells $/ \mathrm{cm}^{2}$, respectively. The detailed method of culturing neurons was described previously (Kohara et al., 2001). All experiments were performed $14-21 \mathrm{~d}$ after plating. In part of the experiments, anti-BDNF antibody ( $30 \mu \mathrm{g} / \mathrm{ml}$; Promega, Madison, WI) was added to the culture medium at $2 \mathrm{~d}$ after plating to neutralize endogenous BDNF.

Injection of plasmid cDNA of DsRed or Neurobiotin. In part of the experiments, plasmid cDNAs of DsRed (DsRed-Express; Clontech, Palo Alto, CA) were injected into the nucleus of neurons through micropipettes at the concentration of $1 \mu \mathrm{g} / \mu \mathrm{l}$ to trace axons of the neurons under observation. Axon terminals of injected neurons were visualized, as reported previously (Kohara et al., 2001). In another series of the experiments, $10 \% \mathrm{~N}$-(2-aminoethyl)biothinamide hydrochloride (Neurobiotin;Vector Laboratories, Burlingame, CA) in PBS was injected into neurons through micropipettes. Neurobiotin was visualized by NeutrAvidin conjugated Alexa 350 (1: 1000; Molecular Probes, Eugene, OR).

Immunocytochemistry. For immunocytochemical staining, neurons were fixed usually with $4 \%$ paraformaldehyde (PFA; Sigma, St. Louis, $\mathrm{MO}$ ) and $4 \%$ sucrose in PBS, pH 7.0, for $30 \mathrm{~min}$ at room temperature. For analysis of dendritic morphology, neurons were fixed with $4 \%$ PFA and $4 \%$ sucrose in PBS, pH 7.4, for 20 min at room temperature. The cells were incubated with PBS containing $0.2 \%$ Triton-X (Sigma) for $1 \mathrm{~min}$ and blocked by $10 \%$ goat serum in PBS for $1 \mathrm{hr}$ at $37^{\circ} \mathrm{C}$. Then, anti-MAP2 monoclonal antibody (isotype:IgG1, 1:250; Sigma), anti-synaptotagmin monoclonal antibody (isotype:IgG2b, 1:200; Calbiochem, San Diego, CA), anti-GAD65 monoclonal antibody (isotype:IgG2a, 1:1000; Chemicon, Temecula, CA), anti-BDNF rabbit polyclonal antibody $(2 \mu \mathrm{g} / \mathrm{ml}$; provided by Dr. R. Katoh-Semba, Institute for Developmental Research, Kasugai 480-0392, Japan) (Katoh-Semba et al., 1997, 2001), anti-GFP chicken polyclonal antibody (1:1000; Chemicon), or anti-GFP rabbit polyclonal antibody (1:1000; Molecular Probes) was applied for $2 \mathrm{hr}$ at $37^{\circ} \mathrm{C}$. MAP2, synaptotagmin, and GAD65 were visualized by isotypespecific secondary antibody conjugated with Alexa 350 (1:200; Molecular Probes), Alexa 546 (1:2000), and Alexa 647 (1:200). GFP and BDNF were visualized by anti-chicken secondary antibody conjugated with Alexa 488 (1:1000) and anti-rabbit secondary antibody conjugated with Cy5 (1: 200; Chemicon). GFP has its own fluorescence but it is gradually fading during observation. Once GFP is immunocytochemically stained with anti-GFP antibody, however, the fluorescence does not fade so quickly. Therefore, immunocytochemical staining of GFP was necessary for observation of neurites of neurons. Fluorescent signals were observed with a $40 \times / 1.3$ numerical aperture oil immersion objective (Plan Flour; Nikon, Tokyo, Japan) attached to an inverted epifluorescence microscope and captured by a cooled CCD camera (C4742-95; Hamamatsu Photonics, Hamamatsu, Japan). This system consisted of $1024 \times 1024$ pixels, each of which corresponded to $0.17 \times 0.17 \mu \mathrm{m}$ with the $40 \times$ objective.
Filters (UV2EC, B2EC, and G2EC; Nikon; XF110; Omega Optical, Brattleboro, VT) were used for four-color immunofluorescence detection. Fluorescence data were analyzed further with an Aquacosmos system (Hamamatsu Photonics). In part of the experiments, a confocal fluorescence microscope (E600FN; Nikon; Radiance 2000; Bio-Rad, Hercules, CA) was used to obtain thin-sliced images of stained neurons.

Measurement of fluorescent signal. The fluorescence intensity of BDNF was measured on a square window $(30 \times 30$ pixels; $5.1 \times 5.1 \mu \mathrm{m})$ placed on the soma of a neuron under observation, and the mean fluorescence intensity of 900 pixels was calculated by subtracting the noise of the CCD camera system that was detected in the complete darkness. The window was placed randomly on the soma in the blind condition, i.e., the window was placed by an experimenter who had not seen any BDNF image of the neuron, and then the fluorescence intensity was measured automatically by the Aquacosmos system. As control, the soma of another neuron that was not contacted by GFP-positive terminals was randomly selected in the same culture dish. The intensity of fluorescence in such a control neuron was calculated in the same way as above. Because the soma of control neurons had some background fluorescence, its fluorescence intensity was expressed as $100 \%$, and that of test neurons was normalized to this value.

Analysis of morphology. After immunocytochemical staining, neurons, the dendrites of which did not overlap with dendrites of other neurons, were randomly selected from the same dishes. After having recorded fluorescent images, neurons were incubated with anti-mouse IgG1conjugated biotin for $1 \mathrm{hr}$ at $37^{\circ} \mathrm{C}$. Then, an $\mathrm{ABC}$ kit (Vector Laboratories) was used for visualization of MAP2. Neurolucida (MicroBrightField, Williston, VT) attached to an upright microscope (E600; Nikon) was used for drawing dendrites of neurons. The quantitative assessment of dendritic morphology was done with an analyzing software, Neuroexplore (MicroBrightField).

\section{Results}

\section{Transfer of endogenous BDNF from presynaptic terminals to postsynaptic neurons}

We prepared chimera culture of cortical neurons from homozygously knock-out mice of BDNF gene (BDNF-/- mice) and from GFP mice (Fig. $1 A$ ). In this culture, GFP-positive neurons are expected to have the potential to express endogenous BDNF, whereas GFP-negative neurons are not. In fact, we confirmed that the former neurons expressed endogenous BDNF in a punctuated manner in neurites (Fig. $1 B, C$ ), whereas the latter neurons did not have any sign of endogenous BDNF (Fig. $1 E, F$ ). Such a GFP-negative neuron became visible by immunocytochemistry with antibody to MAP2, which is a marker of somatodendritic region of neurons (Fig. $1 G$ ).

To identify GABAergic neurons, we stained neurons with antibody to GAD65, which is known to be a synthesizing enzyme of GABA in presynaptic terminals (Erlander and Tobin, 1991). With this immunocytochemistry, we could unambiguously detect GABAergic neurons. Then, we found that endogenous BDNF transferred from presynaptic terminals to postsynaptic GABAergic neurons. An example of this finding is shown in Figure 2. As shown in Figure $2 A$, axon branches of a GFP-positive neuron $(B D N F+/+)$, the soma of which was outside the frame of this picture, made a net-like terminal arborization, which surrounded and contacted a MAP2-positive neuron (red). This postsynaptic neuron was confirmed to be a GABAergic neuron, because the immunoreactivity to antibody against GAD65 was clearly seen in its cell body (Fig. $2 B$, arrowhead). As shown in Figure $2 A$, this neuron was GFP negative and, thus, judged as derived from a BDNF knock-out mouse. In Figure $2 B$, neurites containing GAD65-positive puncta or varicosities were seen around the soma of the GABAergic neuron. These neurites were judged to be axon branches of this and other GABAergic neurons, because they were not stained with anti-MAP2 antibody (Fig. $2 A)$. The superimposed picture in Figure $2 C$ indicates that GFP- 
A
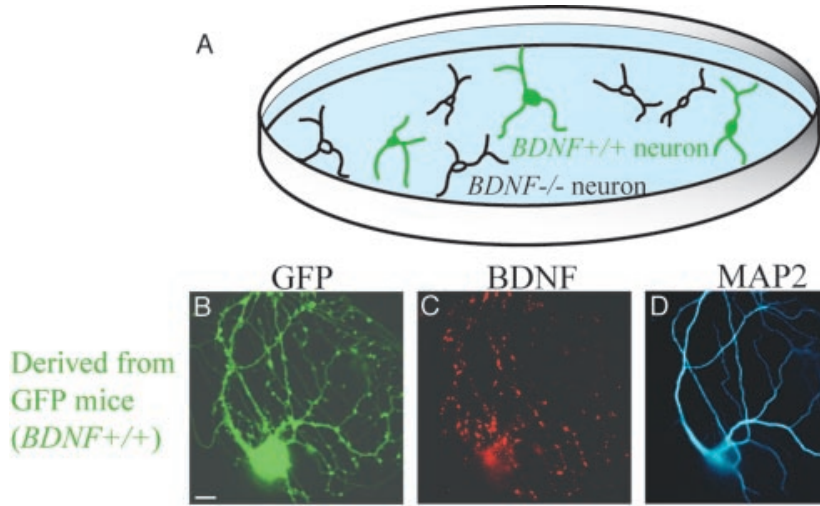

BDNF
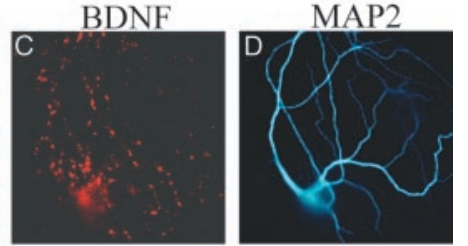

Derived from
$B D N F-/$ - mice

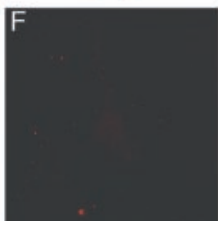

G

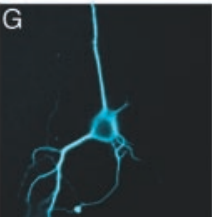

Figure 1. Chimera cell culture prepared from two types of transgenic mice. A, Schematic illustration of chimera culture of cortical neurons derived from BDNF- $/-$ and GFP mice. The latter neurons were labeled with GFP and had the potential to express endogenous BDNF $(B D N F+/+)$. B, GFP image of a cortical neuron derived from a GFP mouse. C, Immunocytochemical BDNF image of the neuron shown in $B . D, M A P 2$ image of the neuron shown in $B$ and $C$. $E$, No GFP fluorescence signal in a cortical neuron derived from a BDNF- / - mouse was observed. This neuron was located in the same dish as above. $F$, No BDNF immunoreactivity in the neuron shown in $E$ was observed. G, MAP2 image of the neuron shown in $E$ and $F$. Scale bar: (in B) $B-G, 10 \mu \mathrm{m}$.

positive axon branches were GAD65 negative and, thus, not GABAergic. In Figure $2 D, E$, it is seen that BDNF-positive puncta were located in presynaptic axon branches. Also, we found that the soma of the GABAergic neuron contained BDNF (Fig. 2D,E, arrows). This suggests that endogenous BDNF was transferred from the presynaptic, GFP-positive axon branches to the postsynaptic GABAergic neuron, because this postsynaptic neuron was derived from a $B D N F-/-$ mouse and, thus, should not produce endogenous BDNF by itself.

There is a possibility that BDNF signals that were detected in the soma region of neurons might be located in the pericellular space that was foreground or background of the soma. To minimize such a possibility, a very thin section $(1.2 \mu \mathrm{m}$ thick $)$ of image was obtained with a confocal microscope in part of the experiments. As indicated by arrows in Figure $3 B-D$, BDNF signals were seen in the soma of a postsynaptic neuron. Also, it was seen that the BDNF signals in the soma were not overlapped with GFP signals, whereas those in neurites were mostly overlapped (Fig. 3C,D). These results confirmed that BDNF was present in the soma of the postsynaptic neuron.

To quantify the transfer of BDNF to postsynaptic neurons, the intensity of fluorescence was measured in the soma of the neurons that were contacted by GFP-positive axon terminals (Fig. $2 F$, insets). As control, the soma of another neuron that was not contacted by GFP-positive terminals was randomly selected in the same culture dish. Because the soma of control neurons had some background fluorescence, its fluorescence intensity was expressed as $100 \%$, and that of test neurons was normalized to this value. The mean fluorescence intensity of the somata of 10 GABAergic neurons that were contacted by GFP-positive axons was $135.0 \pm 8.5 \%$ (mean \pm SEM), which was significantly $(p<$ 0.01 ; unpaired $t$ test) larger than that of the seven control neurons (Fig. 2 F). This indicates that the postsynaptic GABAergic neuron contains endogenous BDNF that has probably been transferred from GFP-positive, $B D N F+/+$ presynaptic axons.

There is a possibility that BDNF detected in the soma of GABAergic neurons might be transferred retrogradely from a GFP-positive neuron. To test such a possibility, we visualized 6 GABAergic neurons with the method of direct intranuclear injection of plasmid cDNAs of DsRed fluorescent protein (Kohara et al., 2001) and another 10 GABAergic neurons with the method of intracellular injection of a tracer, Neurobiotin. With either method, we could trace axons of the neurons until their terminals. Because we did not find a notable difference in visualization of neurons between these two methods, the data were combined. Altogether, six neurons were contacted by GFPpositive afferents. Axon terminals of three neurons contacted GFP-positive, excitatory neurons, and the other three did not. Ten GABAergic neurons were not contacted by GFP-positive afferents. Axon terminals of five of these neurons contacted GFPpositive, excitatory neurons, and those of the other five did not. It is to be noted that the former five neurons that had the possibility to receive BDNF only through the retrograde route did not have detectable BDNF signal in their cell bodies. To quantify these results, the background fluorescence intensity of the soma of the former five neurons was compared with that of the latter five neurons that had no possibility to receive BDNF through either route. The mean fluorescence intensity of the former neurons was $95.9 \pm 4.1 \%$ of that of the latter neurons. The difference was insignificant (unpaired $t$ test; $p>0.05$ ). Thus, these results suggest that the possibility of retrograde transport is negligible in our preparations, although we cannot completely exclude such a mechanism.

An intercellular transfer of endogenous BDNF in the anterograde direction was detected also in GAD65-negative neurons that were most likely excitatory neurons. In part of the experiments, neurons were immunocytochemically stained with antibody to phosphate-activated glutaminase, which is glutamatesynthesizing enzyme in the transmitter pool of cortical neurons (Kaneko and Mizuno, 1988). We observed that all of the GAD65negative neurons were reactive to this antibody, indicating that GAD65-negative neurons were glutamatergic neurons. The mean fluorescence intensity of the somata of 16 GAD65-negative neurons that were contacted by GFP-positive axons was $154.8 \pm$ $16.3 \%$ of that of another 14 GAD65-negative neurons that were not contacted by GFP-positive axons. The difference was statistically significant ( $p<0.01$; unpaired $t$ test). These results indicate that presynaptic endogenous BDNF transferred to both inhibitory and excitatory neurons.

Then, we tested whether BDNF actually exists in presynaptic axon terminals. For this, we stained neurons immunocytochemically with antibody against a presynaptic marker protein, synaptotagmin (Fig. $4 E$ ). Some BDNF signals were localized in GFPpositive axons, so that they appeared to be yellow puncta (Fig. 4C, arrows). As shown in Figure $4 F$, such clusters of BDNF-positive puncta were mostly colocalized with synaptotagmin-positive presynaptic terminals.

\section{Promoted growth of dendrites of GABAergic neurons by presynaptic BDNF}

To assess actions of endogenous BDNF on postsynaptic GABAergic neurons, we quantitatively analyzed the dendritic morphology of neurons that were derived from $B D N F-/-$ mice. As shown in Figure 5, the dendritic morphology of neurons that were immunoreactive to anti-GAD65 antibody (Fig. $5 B, E$ ) was visualized by immunocytochemistry with anti-MAP2 antibody 
(Fig. 5A,D). The neuron shown in Figure $5 A$ was contacted by GFP-positive $(B D N F+/+)$ axon terminals, as shown by yellow puncta in Figure $5 C$, in which the picture stained with anti-GFP antibody (green) was superimposed with that with anti-synaptotagmin antibody (red). The neuron shown in Figure 5D was contacted by GFP-negative axon terminals that were shown by synaptotagmin-postive puncta $(F)$. In Figure $5 A, D$, it is obvious that the GABAergic neuron contacted by GFPpositive $(B D N F+/+)$ terminals had much more abundant dendritic branches than the other neuron contacted by GFPnegative axons $(B D N F-/-)$. The total picture of dendrites of another pair of GABAergic neurons is shown in Figure $6 \mathrm{~A}$. Again, it is obvious that the neuron contacted by GFP-positive $(B D N F+/+)$ terminals had well developed dendrites (right), whereas that contacted by GFPnegative axons (BDNF-/-) had relatively poor dendrites (left).

To quantify this finding, we calculated three parameters of dendritic morphology of each neuron (Fig. 7). The mean total length of dendrites of 14 GABAergic neurons that were contacted by GFP-positive axons $(B D N F+/+)$ and that of other 12 GABAergic neurons that were contacted by GFP-negative axons (BDNF-/-) were $2376.7 \pm 225.5 \mu \mathrm{m}$ and $1345.2 \pm 152.9 \mu \mathrm{m}$, respectively (Fig. $7 A$, left two columns). The difference between these two values was statistically significant ( $p<0.01$; ANOVA). The mean numbers of dendritic branch points of the neurons contacted by GFPpositive and GFP-negative axons were $22.2 \pm 2.0$ and $13.3 \pm 1.9$, respectively (Fig. $7 B$, left two columns). The difference between these two values was, again, significant ( $p<0.01$; ANOVA). In contrast, the number of primary dendrites was not affected by presynapic BDNF (Fig. 7C, left two columns). The mean numbers of primary dendrites of the neurons contacted by GFPpositive and GFP-negative axons were $7.0 \pm 0.5$ and $6.2 \pm 0.6$, respectively. These results suggest that endogenous BDNF, which probably was supplied from presynaptic terminals, may promote branch formation of dendrites of postsynaptic GABAergic neurons.

To confirm that such an effect of contact with GFP-positive $(B D N F+/+)$, presynaptic terminals was induced by extracellularly released BDNF, we applied anti-BDNF antibody, which blocks function of BDNF, to chimera culture preparations. As shown in Figure $6 B$ (right), a GABAergic neuron cultured with anti-BDNF antibody had relatively poor dendrites even when it was contacted by GFP-positive $(B D N F+/+)$ terminals. The complexity of their dendritic arborization seemed to be about the same as that of another GABAergic neuron that was contacted by GFP-negative axons (BDNF-/-) (Fig. 6B, left). This is seen in the quantitative analysis of the three parameters of dendrites (Fig. $7 A-C$, right two columns). The mean total lengths of dendrites of the two groups of GABAergic neurons that were treated with anti-BDNF antibody were $1177.8 \pm 149.5 \mu \mathrm{m}$ and $1173.9 \pm$ $209.8 \mu \mathrm{m}$, respectively (Fig. 7A, right two columns; $n=8$ and 9, respectively). The mean numbers of dendritic branch points of the two groups of neurons were $13.0 \pm 1.1$ and $12.0 \pm 2.2$, re-
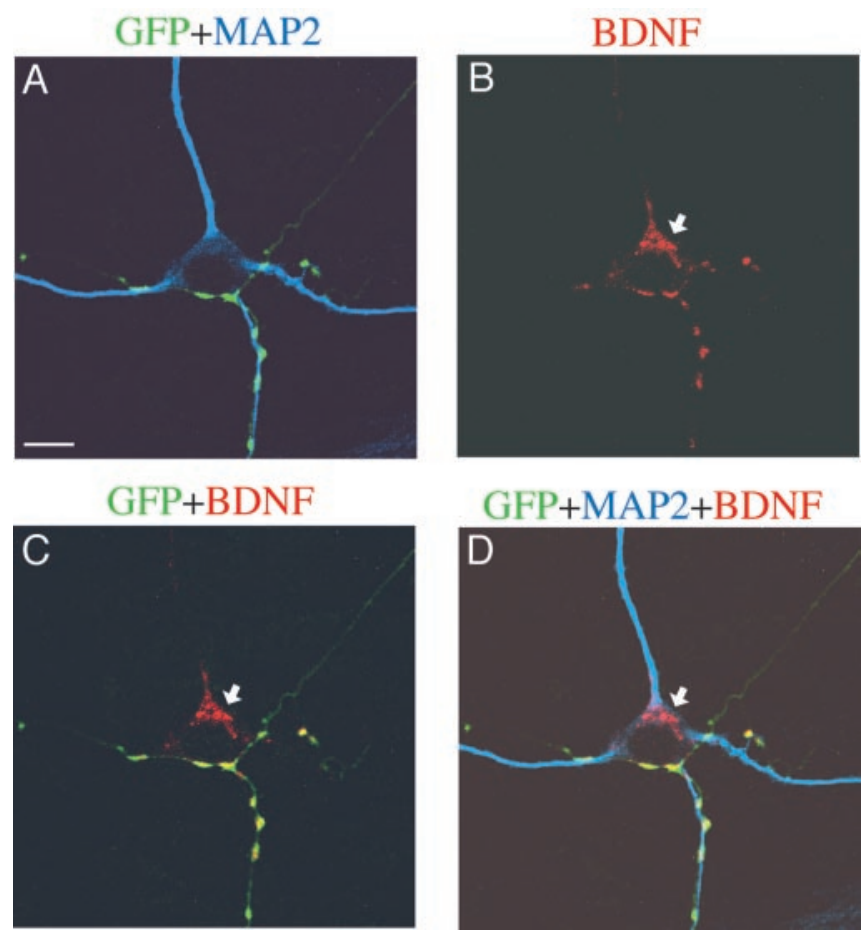

Figure 3. Intraneuronal BDNF visualized by confocal microscopy. All pictures were single focal sections of $1.2 \mu \mathrm{m}$ thickness. A, Superposed image of GFP signals (green) and MAP2 signals (blue). $B, B D N F$ image (red) of the same frame as $A$. The arrow shows BDNF signals in the cell body. C, Superposed image of GFP and BDNF. BDNF signal in the cell body (arrow) was not superposed with GFP-positive neurites. D, Superposed image of GFP, MAP2, and BDNF. Scale bar: (in $A) 10 \mu \mathrm{m}$. 
GFP+MAP2

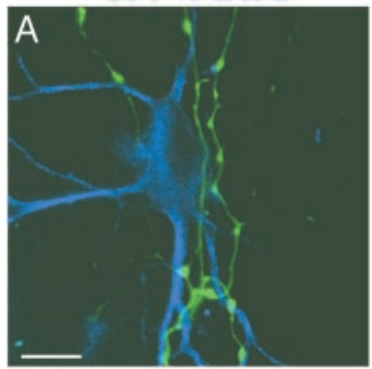

GFP+MAP2+BDNF

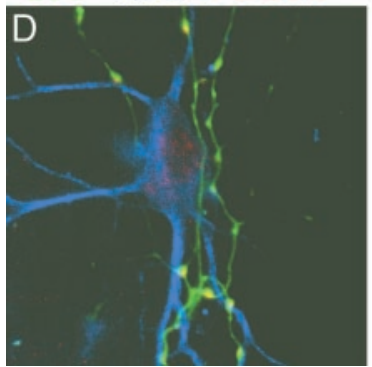

BDNF

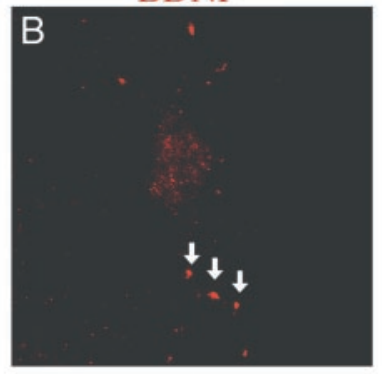

Synaptotagmin

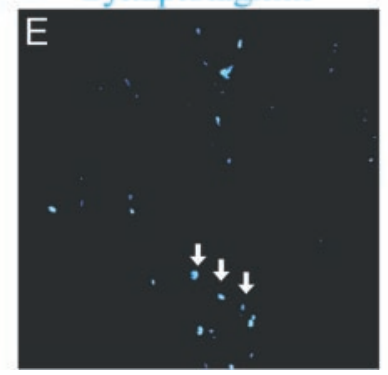

GFP+BDNF

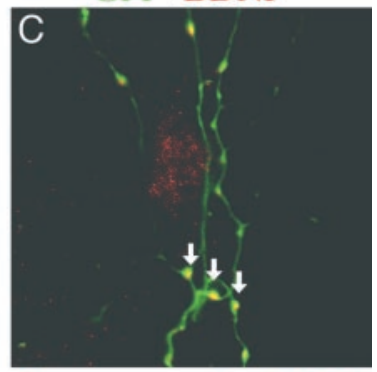

Synaptotagmin+BDNF

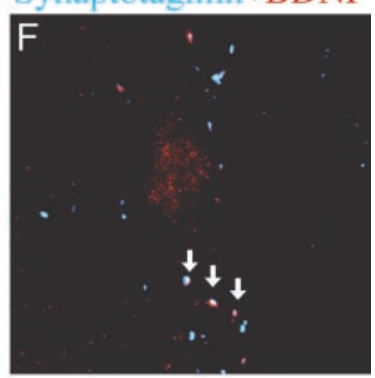

Figure 4. Existence of endogenous BDNF in presynaptic axon terminals. $A$, Superposed image of GFP signals (green) and MAP2 signals (blue). $B, B D N F$ image (red) of the same frame as $A$. The arrows show clusters of BDNF signals. Faint BDNF signal was seen in the soma of the postsynaptic neuron, as shown in D. C, Superposed image of GFP and BDNF. BDNF puncta were colocalized with GFP-positive neurites (arrows). D, Superposed image of GFP, MAP2, and BDNF. E, Synaptotagmin image of the same frame as the others. The arrows indicate the same puncta as in $B, C$, and F.F, Superposed image of synaptotagmin (blue) and BDNF (red). BDNF puncta indicated by arrows were colocalized with synaptotagmin. Scale bar: (in $A$ ) $10 \mu \mathrm{m}$.

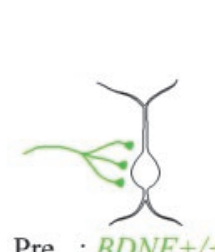

Post : $B D N F-/-$
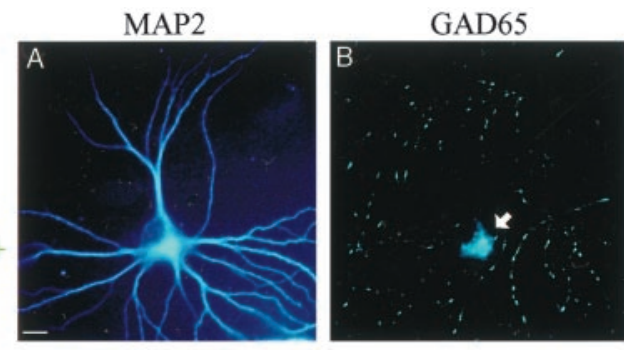

GFP+Synaptotagmin

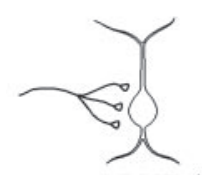

Pre : $B D N F-/-$ Post : $B D N F-/-$
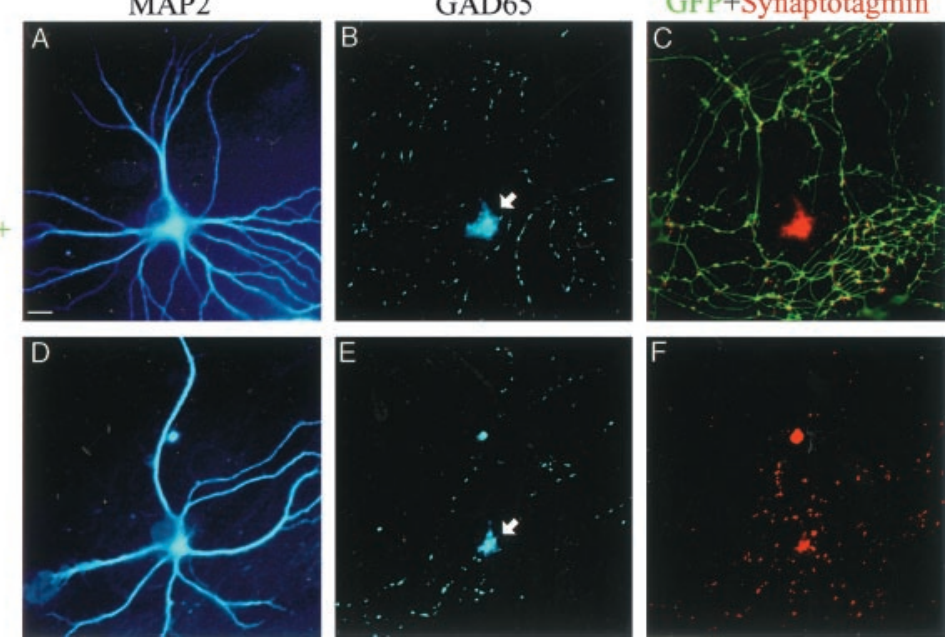

Figure 5. Promoted growth of dendrites of GABAergic neurons by presynaptic BDNF. $A, M A P 2$ image of a neuron contacted by a GFP-positive axon (BDNF+/ + ), as schematically shown on the left. $B, G A D 65$ image of the same frame as in $A$. The arrow shows the cell body. C, Superposition of GFP images (green) and synaptotagmin images (red) of the same frame as $A$ and B.D, MAP2 image of a neuron contacted by a GFP-negative axon (BDNF-/-), as schematically shown on the left. E, GAD65 image of the same frame as in $D$. The arrow shows the cell body. F, Superposition of GFP images (green) and synaptotagmin images (red) of the same frame as $D$ and $E$. Because both the presynaptic and postsynaptic neurons were GFP negative, there was no green signal. Scale bar: (in $A), 10 \mu \mathrm{m}$.

spectively (Fig. $7 B$, right two columns). The mean numbers of primary dendrites of the two groups of neurons were $6.1 \pm 0.5$ and $5.4 \pm 0.7$, respectively (Fig. $7 C$, right two columns). In any of these three parameters, there was no significant difference between the two groups of GABAergic neurons, to which antiBDNF antibody was applied.

In this quantitative analysis, it is also seen that the total length of dendrites and the number of dendritic branch points of the
GABAergic neurons that were contacted by GFP-negative afferents were not reduced by the treatment with the antiBDNF antibody (Fig. $7 A, B$, compare the third columns with the leftmost columns). There was no statistically significant difference (unpaired $t$ test; $p>0.05$ ) between these two columns in any of the three parameters (Fig. 7).

\section{No effect of presynaptic BDNF on} dendritic growth of excitatory neurons

Finally, we examined effects of presynaptic BDNF on the dendritic morphology of GAD65-negative neurons and found that the presence or absence of presynaptic BDNF is not related to dendritic growth of such excitatory neurons. Examples of GAD65-negative neurons are shown in Figure 8 . The GAD65 negativity was obvious, in particular, in their somata (Fig. $8 B, E$, arrows), although the neuron in Figure $8 B$ seemed to be surrounded by GAD65-positive puncta that were assumed to be GABAergic axon terminals. These neurons were judged as derived from $B D N F-/-$ mice, because they were GFP negative (Fig. 8C,F). The seemingly yellow staining of some regions of the neuron shown in Figure $8 F$ was because of GFPpositive presynaptic terminals, the soma of which was outside the frame of this picture. As seen in Figure $8 A, D$, the extent of dendritic arborization of the BDNF-negative excitatory neuron contacted by a GFP-positive axon $(B D N F+/+)($ Fig. $8 D)$ was about the same as that of the neuron contacted by a GFP-negative axon (BDNF-/-) (Fig. 8A).

The dendritic morphology of these two groups of excitatory neurons that were derived from $(B D N F-/-)$ mice was analyzed in the same way as in Figure 7 (Fig. $8 G-I)$. The total length of dendrites of 17 excitatory neurons contacted by GFPpositive axons $(1181.5 \pm 123.1 \mu \mathrm{m})$ was not significantly (unpaired $t$ test; $p>0.05$ ) different from that of another 16 excitatory neurons contacted by GFP-negative axons $(998.1 \pm 120.0 \mu \mathrm{m})$ (Fig. $8 G)$. The mean numbers of dendritic branch points $(11.1 \pm 1.6)$ and primary dendrites $(4.6 \pm$ 0.3 ) of the excitatory neurons with GFPpositive axons were not significantly different from those of the neurons with GFP-negative axons $(10.1 \pm 1.5$ and $4.9 \pm$ 0.5 , respectively) (Fig. $8 H, I$ ) (unpaired $t$ test; $p>0.05$ ). Thus, the presence or absence of presynaptic BDNF had no effects on dendritic development of postsynaptic, excitatory neurons.

\section{Discussion}

It is well established that GABAergic neurons do not have mRNA of BDNF and, thus, cannot produce BDNF by themselves (Ernfors et al., 1990; Cellerino et al., 1996; Rocamora et al., 1996; 
A Contacted by $B D N F-/$ terminals

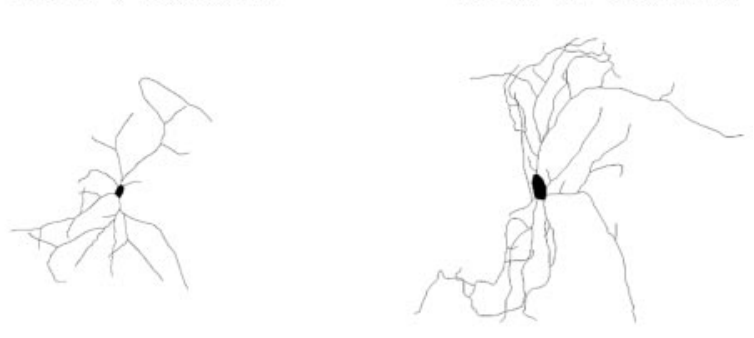

+ BDNF antibody

B

Contacted by

$B D N F$-/- terminals

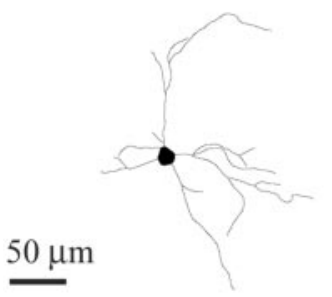

Contacted by $B D N F+/+$ terminals

Contacted by

$B D N F+/+$ terminals

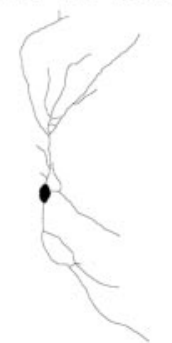

Figure 6. Morphology of dendrites of GABAergic neurons and effects of anti-BDNF antibody. $A$, Left, Dendritic morphology of a GABAergic neuron (BDNF-/-) that was contacted by GFPfree $(B D N F-/-)$ axons. Right, Dendritic morphology of another GABAergic neuron (BDNF-/-) that was contacted by GFP-positive (BDNF+/+) axons. B, Dendritic morphology of GABAergic neurons (BDNF- - ) treated with anti-BDNF antibody. Left and right, Neurons were contacted by GFP-free axons (BDNF-/-) and GFP-positive axons $(B D N F+/+)$, respectively.

Gorba and Wahle, 1999). In the present study, we have found that such GABAergic neurons had a clear signal of the existence of BDNF in their cell bodies. This BDNF is judged to have been transferred from presynaptic GFP-positive axons that contain endogenous BDNF, because only the GABAergic neurons that were contacted by GFP-positive axon terminals had significant signals, but those that were not contacted by such terminals had not. A previous report also suggested that interneurons could incorporate BDNF that might be released from pyramidal cells in rat hippocampus (Schmidt-Kastner et al., 1996). However, they did not show that their interneurons were GABAergic and also did not provide direct evidence that BDNF was actually transferred to those neurons from pyramidal neurons.

The present study further indicates that BDNF that has been transferred in such a way promotes dendritic development of GABAergic neurons, because the neurons contacted by GFPpositive axon terminals had well developed dendrites, but those not contacted by such terminals had relatively poor dendrites. The better development of dendrites of the former neurons is thought to result from intercellularly transferred BDNF, because anti-BDNF antibody that was applied through the culture medium and, thus, expected to neutralize BDNF in the extracellular space blocked such a proliferous action.

There is a possibility that GABAergic neurons might receive BDNF through their axons from GFP-positive, postsynaptic neurons and such target-derived BDNF might exert the proliferous action, because BDNF is known to be transported also retro-
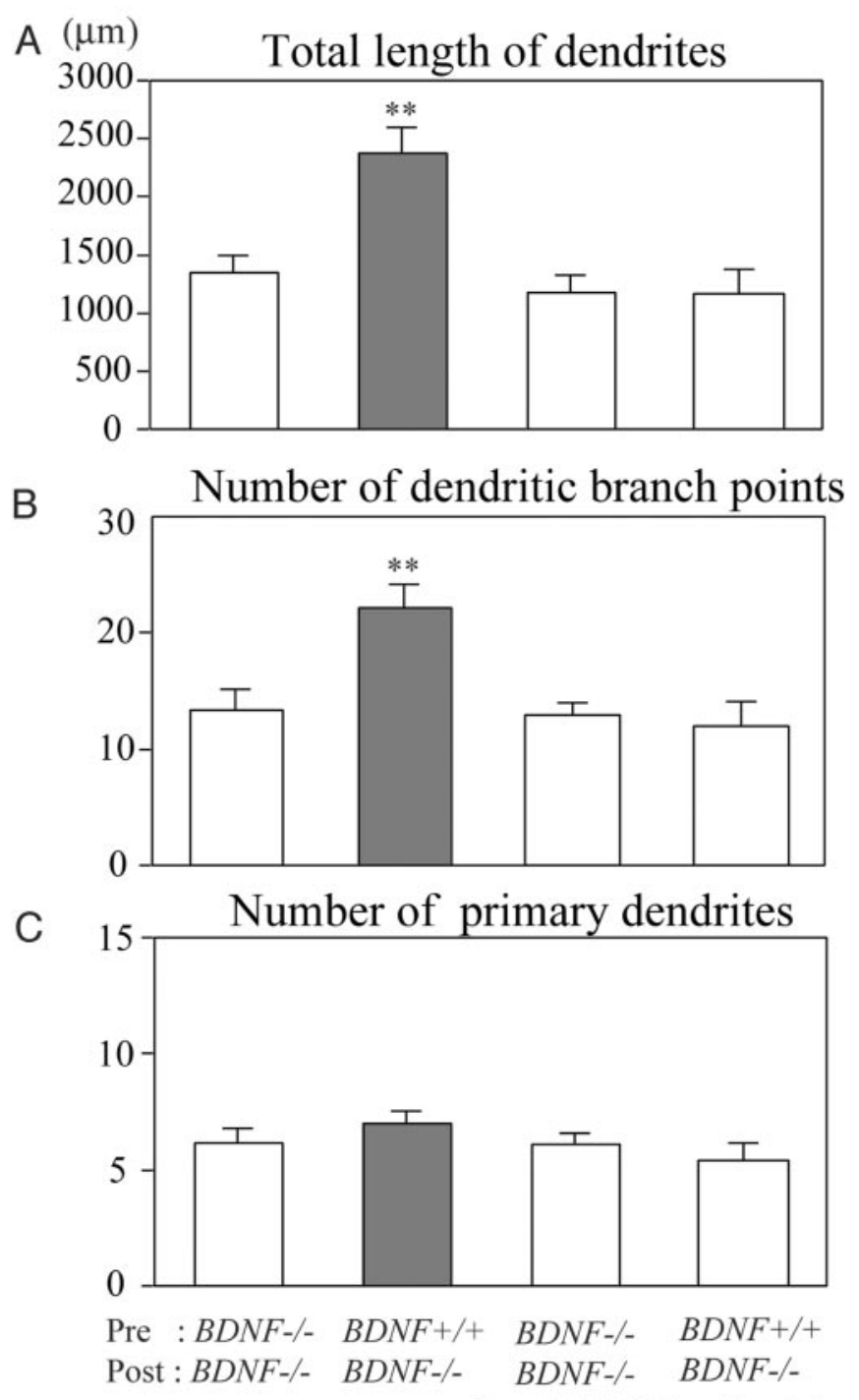

(+anti-BDNF antibody)

Figure 7. Quantitative assessment of actions of presynaptic BDNF on dendrites of GABAergic neurons. $A$, Mean values of the total length of dendrites of neurons contacted by GFP-negative axons (BDNF-/-) (leftmost column) and GFP-positive axons (BDNF $+/+$ ) (hatched column) in the control medium and corresponding values of neurons treated with anti-BDNF antibody (third and rightmost columns). Vertical bars indicate SEM. Double asterisks in $A$ and $B$ indicate statistical significance of the difference at $p<0.01$ (ANOVA). $B$, Mean number of dendritic branch points per neuron. $C$, Mean number of primary dendrites per neuron. In $B$ and $C$, neurons are grouped in the same way as in $A$.

gradely from postsynaptic neurons to presynaptic axon terminals (Causing et al., 1997; Marty et al., 1997; Watson et al., 1999). This possibility seems unlikely, however, for two reasons. First, in the present study, none of the GABAergic neurons that did not receive GFP-positive afferents but sent their axons to other GFPpositive, excitatory neurons had detectable BDNF signal in their cell bodies. If endogenous BDNF is transferred in the retrograde direction, we should have detected BDNF signal in such GABAergic neurons. Second, if target-derived BDNF has some effects on GABAergic neurons, the functional block of released BDNF by the anti-BDNF antibody should have affected dendritic development of the GABAergic neurons that were contacted by GFPnegative terminals. The results shown in Figure 7 indicate, however, that the application of the antibody did not exert significant 


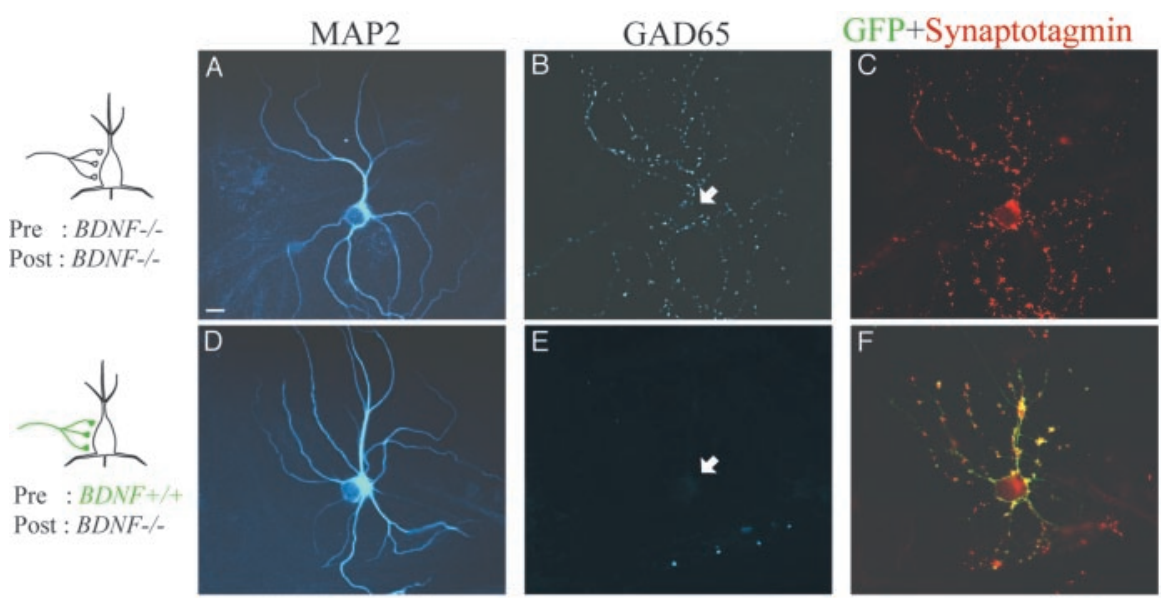

$\mathrm{G}$ Total length of dendrites $\mathrm{H}$ Number of branch points
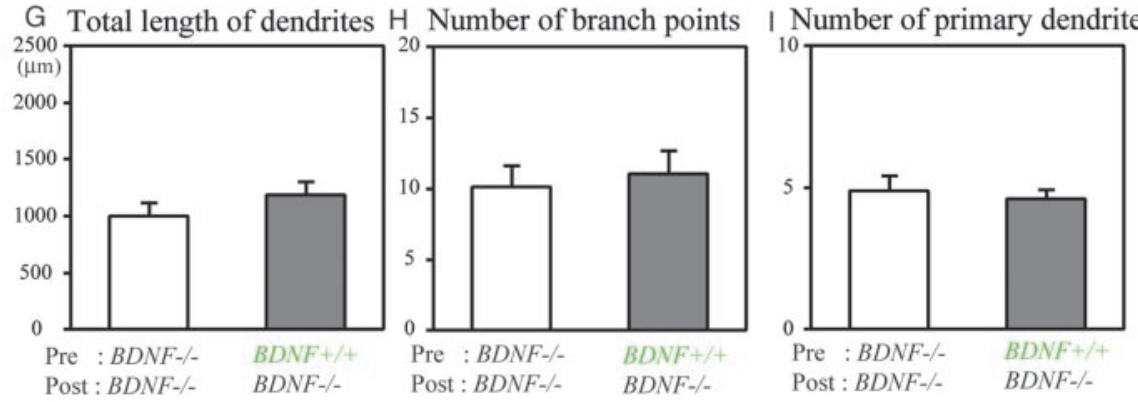

Figure 8. No effect of presynaptic BDNF on growth of dendrites of excitatory neurons. $A, D, M A P 2$ images of a BDNF-/neuron that was contacted by $B D N F-/-$ axons and $B D N F+/+$ axons, respectively. $B, E, G A D 65$ images of the neurons shown in $A$ and $D$, respectively. The arrows indicate the location of the soma. Note the lack of label in the cell body of both neurons. $C, F$, Superposed image of GFP and synaptotagmin signals of the neurons shown in $A$ and $D$, respectively. Scale bar: (in $A) 10 \mu \mathrm{m}$. $G$, Mean value of the total length of dendrites of excitatory neurons contacted by GFP-negative axons (BDNF- $/-$ ) (open column) and by GFP-positive axons (BDNF+/+) (hatched column). Vertical bars indicate SEM. $H$, Mean number of branch points of dendrites per neuron. I, Mean number of primary dendrites per neuron.

actions on the dendritic development of this group of GABAergic neurons (Fig. $7 A-C$, compare the third with leftmost columns).

In the present chimera culture preparations, the densities of GFP/BDNF-positive and -negative neurons were different. If the density of the former neurons was higher than that of the latter neurons, the number of GFP/BDNF-positive terminals might have exceeded that of GFP/BDNF-negative terminals. Consequently, GABAergic neurons that were contacted by the former terminals might have developed their dendrites better simply because of the greater number of contacts, irrespectively of BDNF transfer. To minimize this possibility, we set the ratio of the density of GFP/BDNF-positive and -negative cells at 1:20-40. In this condition, the number of GFP/BDNF-positive terminals was much less than that of negative terminals. Nevertheless, GFP/ BDNF-positive terminals exerted the promoting action on dendritic growth of postsynaptic GABAergic neurons. Therefore, the possibility mentioned above seems unlikely in the present preparations.

Because GABAergic neurons do not have mRNA of BDNF as mentioned previously, the transfer of BDNF from excitatory neurons may be crucial for development of dendritic arborization of GABAergic neurons. In visual cortex in vivo, it is known that most, if not all, GABAergic neurons are contacted by axons of pyramidal neurons (Kisvarday, 1992; Johnson and Burkhalter, 1996). Also, it is well established that neocortical pyramidal neurons express BDNF (Yan et al., 1997; Friedman et al., 1998). Thus, it seems reasonable to assume that endogenous BDNF released from pyramidal cell axons acts on GABAergic neurons in visual cortex in vivo. However, previous studies in hippocampal neurons suggested that BDNF transferred retrogradely from excitatory neurons to GABAergic neurons may promote growth of the latter neurons (Marty et al., 1996, 1997). The present results have demonstrated, however, that the anterograde transfer of BDNF from excitatory neurons to GABAergic neurons plays such a role, although we cannot exclude any retrograde transfer. Anterogradely transferred BDNF, then, may activate the local protein synthesis that leads to growth of dendrites of postsynaptic neurons, as suggested (Aakalu et al., 2001; Takei et al., 2001).

In excitatory neurons, the transcellular transfer of BDNF seems to be not so important in the development of their dendrites, because the dendritic development was not correlated with the existence of presynaptic BDNF. This raises a possibility that the expression of functionally active or inactive BDNF receptors such as fulllength and truncated TrkB in excitatory neurons might be different from that of inhibitory neurons. To our knowledge, there was no study in which the two types of receptors were differentially stained, except for a study in which full-length TrkB and pan TrkB including truncated type were differentially stained in the hippocampal formation of adult rats (Drake et al., 1999). In this study, no marked difference was reported in localization of immunoreactivity between full-length and pan TrkBs, although the intensity of reactivity was different at various subcellular sites. Thus, a reason why transferred BDNF was less important in dendritic development of excitatory neurons is not clear in the present study. Because excitatory neurons can produce BDNF by themselves, however, it is to be noted that endogenous BDNF may act on this type of neurons, in part at least, through an autocrine loop, as suggested previously (Kokaia et al., 1993; Miranda et al., 1993; Horch et al., 1999). From the present results, it is possible to suggest that the different actions of BDNF on excitatory and inhibitory synapses that were reported previously (Rutherford et al., 1998; Schinder et al., 2000) may be executed through the distinct pathways (i.e., anterograde, transsynaptic route to GABAergic neurons and autocrine route to glutamatergic neurons).

Because the release and transcellular transfer of BDNF are known to depend on neuronal activity (Goodman et al., 1996; Balkowiec and Katz, 2000; Hartmann et al., 2001; Kohara et al., 2001; Kojima et al., 2001; Lever et al., 2001; Gartner and Staiger, 2002), the transfer of BDNF to GABAergic neurons is assumed to be activity dependent. It is suggested that maturation of GABAergic neurons regulates the beginning of the critical period during which visual cortical neurons are highly sensitive to an alteration in inputs (Hensch et al., 1998). Furthermore, an overexpression of BDNF induces a precocious critical period in mouse visual cortex probably through its action on GABAergic neurons (Hanover et al., 1999; Huang et al., 1999). Thus, it is possible to suggest that the activity-dependent transfer of endogenous 
BDNF to GABAergic neurons may promote their maturation so as to play a role in the onset of the critical period of the developing visual cortex.

It was reported that another neurotrophin, NT-3, abolished the growth-promoting effect of BDNF on pyramidal neurons in slice culture preparations of visual cortex of young ferrets (McAllister et al., 1997). A question of whether NT-3 and BDNF have such an antagonist action also on GABAergic neurons should be addressed in a future study. Finally, it is to be noted that chimera culture preparations of neurons derived from different kinds of transgenic mice are a useful tool to elucidate the functional significance of a given bioactive molecule. Although conditional knock-out systems using Cre-loxP and specific promoter have been developed to reveal the local functions of target proteins (Tsien et al., 1996; Minichiello et al., 1999; Xu et al., 2000a,b; Iwasato et al., 2000), it is difficult to delete them from particular synapses of given neuronal circuits. In chimera cultures of neurons, in contrast, it is easy to make neuronal circuits in which particular molecules are lacking in visually identifiable circuits. In fact, we have successfully demonstrated that dendritic development of inhibitory cortical neurons is regulated by presynaptic BDNF.

\section{References}

Aakalu G, Smith WB, Nguyen N, Jiang C, Schuman EM (2001) Dynamic visualization of local protein synthesis in hippocampal neurons. Neuron 30:489-502.

Altar CA, Cai N, Bliven T, Juhasz M, Conner JM, Acheson AL, Lindsay RM, Wiegand SJ (1997) Anterograde transport of brain-derived neurotrophic factor and its role in the brain. Nature 389:856-860.

Balkowiec A, Katz DM (2000) Activity-dependent release of endogenous brain-derived neurotrophic factor from sensory neurons detected by ELISA in situ. J Neurosci 20:7417-7423.

Bartrup JT, Moorman JM, Newberry NR (1997) BDNF enhances neuronal growth and synaptic activity in hippocampal cell cultures. NeuroReport 8:3791-3794.

Bibel M, Barde YA (2000) Neurotrophins: key regulators of cell fate and cell shape in the vertebrate nervous system. Genes Dev 14:2920-2935.

Bolton MM, Pittman AJ, Lo DC (2000) Brain-derived neurotrophic factor differentially regulates excitatory and inhibitory synaptic transmission in hippocampal cultures. J Neurosci 20:3221-3232.

Causing CG, Gloster A, Aloyz R, Bamji SX, Chang E, Fawcett J, Kuchel G, Miller FD (1997) Synaptic innervation density is regulated by neuronderived BDNF. Neuron 18:257-267.

Cellerino A, Maffei L, Domenici L (1996) The distribution of brain-derived neurotrophic factor and its receptor trkB in parvalbumin-containing neurons of the rat visual cortex. Eur J Neurosci 8:1190-1197.

Drake CT, Milner TA, Patterson SL (1999) Ultrastructural localization of full-length trkB immunoreactivity in rat hipppcampus suggests multiple roles in modulating activity-dependent synaptic plasticity. J Neurosci 19:8009-8026

Erlander MG, Tobin AJ (1991) The structural and functional heterogeneity of glutamic acid decarboxylase: a review. Neurochem Res 16:215-226.

Ernfors P, Wetmore C, Olson L, Persson H (1990) Identification of cells in rat brain and peripheral tissues expressing mRNA for members of the nerve growth factor family. Neuron 5:511-526.

Fawcett JP, Alonso-Vanegas MA, Morris SJ, Miller FD, Sadikot AF, Murphy RA (2000) Evidence that brain-derived neurotrophic factor from presynaptic nerve terminals regulates the phenotype of calbindin-containing neurons in the lateral septum. J Neurosci 20:274-282.

Frerking M, Malenka RC, Nicoll RA (1998) Brain-derived neurotrophic factor (BDNF) modulates inhibitory, but not excitatory, transmission in the CA1 region of the hippocampus. J Neurophysiol 80:3383-3386.

Friedman WJ, Black IB, Kaplan DR (1998) Distribution of the neurotrophins brain-derived neurotrophic factor, neurotrophin-3, and neurotrophin-4/5 in the postnatal rat brain: an immnunocytochemical study. Neuroscience 84:101-114.

Fujikawa N, Tominaga-Yoshino K, Okabe M, Ogura A (2000) Depolarization- dependent survival of cultured mouse cerebellar granule neurons is strainrestrained. Eur J Neurosci 12:1838-1842.

Gartner A, Staiger V (2002) Neurotrophin secretion from hippocampal neurons evoked by long-term potentiation-inducing electrical stimulation patterns. Proc Natl Acad Sci USA 99:6386-6391.

Goodman LJ, Valverde J, Lim F, Geschwind MD, Federoff HJ, Geller AI, Hefti F (1996) Regulated release and polarized localization of brain-derived neurotrophic factor in hippocampal neurons. Mol Cell Neurosci 7:222-238.

Gorba T, Wahle P (1999) Expression of TrkB and TrkC but not BDNF mRNA in neurochemically identified interneurons in rat visual cortex in vivo and in organotypic cultures. Eur J Neurosci 11:1179-1190.

Hanover JL, Huang ZJ, Tonegawa S, Stryker MP (1999) Brain-derived neurotrophic factor overexpression induces precocious critical period in mouse visual cortex. J Neurosci 19:RC40.

Hartmann M, Heumann R, Lessmann V (2001) Postsynaptic release of brain-derived neurotrophic factor induced by high frequency synaptic stimulation. EMBO J 20:5887-5897.

Hensch TK, Fagiolini M, Mataga N, Stryker MP, Baekkeskov S, Kash SF (1998) Local GABA circuit control of experience-dependent plasticity in developing visual cortex. Science 282:1504-1508.

Horch HE, Kruttgen A, Portbury SD, Katz LC (1999) Destablization of cortical dendrites and spines by BDNF. Neuron 23:353-364.

Huang ZJ, Kirkwood A, Pizzorusso T, Porciatti V, Morales B, Bear MF, Maffei L, Tonegawa S (1999) BDNF regulates the maturation of inhibition and the critical period of plasticity in mouse visual cortex. Cell 98:739-755.

Ip NY, Li Y, Yancopoulos GD, Lindsay R (1993) Cultured hippocampal neurons show responses to BDNF, NT-3, and NT-4, but not NGF. J Neurosci 13:3394-3405.

Itami C, Mizuno K, Kohno T, Nakamura S (2000) Brain-derived neurotrophic factor requirement for activity-dependent maturation of glutamatergic synapse in developing mouse somatosensory cortex. Brain Res 857:141-150.

Ivkovic S, Ehrlich ME (1999) Expression of the striatal DARPP-32/ ARPP-21 phenotype in GABAergic neurons requires neurotrophins in vivo and in vitro. J Neurosci 19:5409-5419.

Iwasato T, Datwani A, Wolf AM, Nishiyama H, Taguchi Y, Tonegawa S, Knopfel T, Erzurumlu RS, Itohara S (2000) Cortex-restricted disruption of NMDAR1 impairs neuronal patterns in the barrel cortex. Nature 406:726-731.

Johnson RR, Burkhalter A (1996) Microcircuitry of forward and feedback connections within rat visual cortex. J Comp Neurol 368:383-398.

Kaneko T, Mizuno N (1988) Immunohistochemical study of glutaminasecontaining neurons in the cerebral cortex and thalamus of the rat. J Comp Neurol 267:590-602.

Katoh-Semba R, Takeuchi IK, Semba R, Kato K (1997) Distribution of brain-derived neurotrophic factor in rats and its changes with development in the brain. J Neurochem 69:34-42.

Katoh-Semba R, Takeuchi IK, Inaguma Y, Ichisaka S, Hata Y, Tsumoto T, Iwai M, Mikoshiba K, Kato K (2001) Induction of brain-derived neurotrophic factor by convulsive drugs in the rat brain: involvement of regionspecific voltage-dependent calcium channels. J Neurochem 77:71-83.

Kisvarday ZF (1992) GABAergic networks of basket cells in the visual cortex. Prog Brain Res 90:385-405.

Kohara K, Kitamura A, Morishima M, Tsumoto T (2001) Activitydependent transfer of brain-derived neurotrophic factor to postsynaptic neurons. Science 291:2419-2423.

Kojima M, Takei N, Numakawa T, Ishikawa Y, Suzuki S, Matsumoto T, Katoh-Semba R, Nawa H, Hatanaka H (2001) Biological characterization and optical imaging of BDNF-GFP suggest an activity-dependent local release of BDNF in neurites of cultured hippocampal neurons. J Neurosci Res 64:1-10.

Kokaia ZJ, Bengson M, Matsis M, Kokaia M, Persson H (1993) Coexpression of neurotrophins and their receptors in neurons of the central nervous system. Proc Natl Acad Sci USA 90:6711-6715.

Lever IJ, Bradbury EJ, Cunningham JR, Adelson DW, Jones MG, McMahon SB, Marvizon JC, Malcangio M (2001) Brain-derived neurotrophic factor is released in the dorsal horn by distinctive patterns of afferent fiber stimulation. J Neurosci 21:4469-4477.

Marty S, Berninger B, Carroll P, Thoenen H (1996) GABAergic stimulation regulates the phenotype of hippocampal interneurons through the regulation of brain-derived neurotrophic factor. Neuron 16:565-570. 
Marty S, Berzaghi MD, Berninger B (1997) Neurotrophins and activitydependent plasticity of cortical interneurons. Trends Neurosci 20:198-202.

Marty S, Wehrle R, Sotelo C (2000) Neuronal activity and brain-derived neurotrophic factor regulate the density of inhibitory synapses in organotypic slice cultures of postnatal hippocampus. J Neurosci 20:8087-8095.

McAllister AK, Lo DC, Katz LC (1995) Neurotrophins regulate dendritic growth in developing visual cortex. Neuron 15:791-803.

McAllister AK, Katz LC, Lo DC (1996) Neurotrophin regulation of cortical dendritic growth requires activity. Neuron 17:1057-1064.

McAllister AK, Katz LC, Lo DC (1997) Opposing roles for endogenous BDNF and NT-3 in regulating cortical dendritic growth. Neuron 18:767-778.

McAllister AK, Katz LC, Lo DC (1999) Neurotrophins and synaptic plasticity. Annu Rev Neurosci 22:295-318.

Minichiello L, Korte M, Wolfe D, Kuhn R, Unsicker K, Cestari V, RossiArnaud C, Lipp HP, Bonhoeffer T, Klein R (1999) Essential role for TrkB receptors in hippocampus-mediated learning. Neuron 24:401-414.

Miranda RJ, Sorabji F, Torrand-Allerand D (1993) Neuronal colocaliztion of mRNAs for neurotrophins and their receptors in the developing central nervous system suggests a potential for autocrine interactions. Proc Natl Acad Sci USA 90:6439-6443.

Mizuno K, Carnahan Y, Nawa H (1994) Brain-derived neurotrophic factor promotes differentiation of striatal GABAergic neurons. Dev Biol 165:243-256.

Nawa H, Bessho Y, Carnahan Y, Nakanishi S, Mizuno K (1993) Regulation of neuropeptide expression in cultured cerebral cortical neurons by brain-derived neurotrophic factor. J Neurochem 60:772-775.

Nawa H, Carnahan J, Gall C (1995) BDNF protein measured by a novel enzyme immunoassay in normal brain and after seizure: partial disagreement with mRNA levels. Eur J Neurosci 7:1527-1535.

Okabe M, Ikawa M, Kominami K, Nakanishi T, Nishimune Y (1997) "Green mice" as a source of ubiquitous green cells. FEBS Lett 407:313-319.

Poo MM (2001) Neurotrophins as synaptic modulators. Nat Rev Neurosci 2:24-32.

Rocamora N, Welker E, Pascual M, Soriano E (1996) Upregulation of BDNF mRNA in the barrel cortex of adult mice after sensory stimulation. J Neurosci 16:4411-4419.

Rutherford LC, DeVan A, Lauer HM, Turrigiano GG (1997) Brain-derived neurotrophic factor mediates the activity-dependent regulation of inhibition in neocortical cultures. J Neurosci 17:4527-4535.

Rutherford LC, Nelson SB, Turrigiano GG (1998) BDNF has opposite effects on the quantal amplitude of pyramidal neuron and interneuron excitatory synapses. Neuron 21:521-530.
Schinder AF, Berninger M, Poo MM (2000) Postsynaptic target specificity of neurotrophin-induced presynaptic potentiation. Neuron 25:151-163.

Schmidt-Kastner R, Wetmore C, Olson L (1996) Comparative study of brain-derived neurotrophic factor messenger RNA and protein at the cellular level suggests multiple roles in hippocampus, striatum and cortex. Neuroscience 74:161-183.

Schwartz PM, Borghesani PR, Levy RL, Pomeroy SL, Segal RA (1997) Abnormal cerebellar development and foliation in $\mathrm{BDNF}-/-$ mice reveals a role for neurotrophins in CNS patterning. Neuron 19:269-281.

Takei N, Kawamura M, Hara K, Yonezawa K, Nawa H (2001) Brain-derived neurotrophic factor enhances neuronal translation by activating multiple initiation processes. J Biol Chem 276:42818-42825.

Tanaka T, Saito H, Matsuki N (1997) Inhibition of $\mathrm{GABA}_{\mathrm{A}}$ synaptic responses by brain-derived neurotrophic factor (BDNF) in rat hippocampus. J Neurosci 17:2959-2966.

Thoenen H (1995) Neurotrophins and neuronal plasticity. Science 270:593-598.

Tsien JZ, Chen DF, Gerber D, Tom C, Mercer EH, Anderson DJ, Mayford M, Kandel ER, Tonegawa S (1996) Subregion- and cell type-restricted gene knockout in mouse brain. Cell 87:1317-1326.

Vicario-Abejon C, Collin C, McKay RDG, Segal M (1998) Neurotrophins induce formation of functional excitatory and inhibitory synapses between cultured hippocampal neurons. J Neurosci 18:7256-7271.

Watson FL, Heerssen HM, Moheban DB, Lin MZ, Sauvageot CM, Bhattacharyya A, Pomeroy SL, Segal RA (1999) Rapid nuclear responses to target-derived neurotrophins require retrograde transport of ligandreceptor complex. J Neurosci 19:7889-7900.

Xu B, Gottschalk W, Chow A, Wilson RI, Schnell E, Zang K, Wang D, Nicoll RA, Lu B, Reichardt LF (2000a) The role of brain-derived neurotrophic factor receptors in the mature hippocampus: Modulation of long-term potentiation through a presynaptic mechanism involving TrkB. J Neurosci 20:6888-6897.

Xu B, Zang K, Ruff NL, Zhang A, McConnell SK, Stryker MP, Reichardt LF (2000b) Cortical degeneration in the absence of neurotrophin signaling: Dendritic retraction and neuronal loss after removal of the receptor TrkB. Neuron 26:233-245.

Yamada MK, Nakanishi K, Ohba S, Nakamura T, Ikegaya Y, Nishiyama N, Matsuki N (2002) Brain-derived neurotrophic factor promotes the maturation of GABAergic mechanisms in cultured hippocampal neurons. J Neurosci 22:7580-7585.

Yan Q, Rosenfeld RD, Matheson CR, Hawkins N, Lopez OT, Bennett L, Welcher AA (1997) Expression of brain-derived neurotrophic factor protein in the adult rat central nervous system. Neuroscience 78:431-448. 